\title{
Mycological Profile of Otomycosis in Tertiary Care Teaching Hospital
}

\author{
Wadile Rahul Gopichand* \\ Department of Microbiology, JMF's ACPM Medical Colleg, Dhule - 424001, India \\ *Corresponding author
}

\section{Keywords \\ Otomycosis, Aspergillus, Candida, Mucor, Sabouraud's Dextrose Agar (SDA)}

Article Info

Accepted:

10 June 2019

Available Online:

10 July 2019
A B S T R A C T

Otomycosis is an acute, subacute or chronic fungal infection of the external auditory canal. It is worldwide in distribution with a higher prevalence in the hot, humid, and dusty areas of the tropics and subtropics. A wide variety of fungi can cause Otomycosis. In this study, our aim was to determine the most common mode of presentation, predisposing factors, and the spectrum of fungi involved in Otomycosis. 60 Ear discharge samples were collected aseptically using two sterile cotton swabs. One swab was used for direct microscopic examination and used for fungal culture. For mycological identification, direct microscopic examination was carried out by $10 \% \mathrm{KOH}$ examination and inoculation of material was done on two slants of Sabouraud's Dextrose Agar (SDA) with chloramphenicol (Himedia, India). Identification was done on the basis of colony morphology, Lactophenol Cotton Blue (LPCB) mount microscopy and germ tube test. Out of 60 specimens, Fungal isolates were found in 44 (73.33\%) specimens. Aspergillus niger $21(47.72 \%)$ was the predominant species isolated. Followed by Aspergillus flavus 12 (27.27\%), Candida albicans 05 (11.36\%), Aspergillus fumigatus 04 (9.10\%), Mucor 01 (2.27\%) and Penicillium species 01 (2.27\%) Highest frequency of Otomycosis was found in the age group of $15-35$ years, followed by $>35-55$ years. Predominant predisposing factor for Otomycosis was found to be instillation of coconut oil $(36.36 \%)$ followed by self-cleaning $(31.82 \%)$. Hearing loss $(77.27 \%)$ was the predominant symptom in Otomycotic patients followed by pain $(70.45 \%)$. Rural communities with higher practice of self-cleaning and using home remedies to get relief from ear ailments are at higher risk of Otomycosis. Species like Aspergillus and Candida are the common species involved in such cases. There is a need to educate rural population about serious complications of selfcleaning and using home remedies for ear problems.

\section{Introduction}

Otomycosis is a superficial, sub-acute or chronic infection of the external auditory canal, usually unilateral, that is characterised by inflammation, pruritus and scaling (Agrawal et al., 2001). Otomycosis is worldwide in distribution with prevalence ranging from $9 \%$ to $30 \%$ among patients presenting with signs and symptoms of otitis externa and discharging ears in otolaryngology settings. The frequency of otomycosis depends upon different climatic conditions with higher prevalence in the hot, humid and dusty areas of the tropics and subtropics (Pontes et al., 2009; Pradhan et al., 2003). Most patients suffer from Otomycosis complain of severe itching which often 
progress to pain, hearing loss, and often leading to tympanic membrane perforations (Viswanatha et al., 2012). The most isolated fungi are Aspergillus species and Candida species. (Mishra et al., 2004) However, other fungi can cause Otomycosis. Treatment recommendations go from germ termination or controlling predisposing factors, to local debridement (micro-aspiration) and/or the use of antimicrobial agents (topical/systemic) (Hurst et al., 2001).

The present study was conducted to determine the common presenting symptoms, predisposing factors and spectrum of fungi involved in Otomycosis.

\section{Materials and Methods}

This study was carried out in the Department of microbiology, JMF's ACPM Medical College, Dhule. 44 cases selected for this study were taken from OPD of ENT department of ACPMCH, Dhule. Ear discharge samples were collected aseptically using two sterile cotton swabs. One swab was used for direct microscopic examination and used for fungal culture.

For mycological identification, direct microscopic examination was carried out by $10 \% \mathrm{KOH}$ examination and inoculation of material was done on two slants of Sabouraud's Dextrose Agar (SDA) with chloramphenicol (Himedia, India), which was incubated at $25^{\circ} \mathrm{C}$ and $37^{\circ} \mathrm{C}$ aerobically for a period of 4 weeks. Culture media were examined for presence of colonies every 3-4 days. Identification was done on the basis of colony morphology and Lactophenol Cotton Blue (LPCB) mount microscopy. Aspergillus isolates were characterized by varying length of conidiophores and extent of coverage of vesicles by phialides and conidia. For characterization of Candida isolates, germ tube test was done by observing the production of germ tubes on isolates in serum after 1-2 hours of incubation at $37^{\circ} \mathrm{C}$ and colonies were inoculated on HiChrome agar for identification of species. (Chander $\mathrm{J}$ et al., 2018)

\section{Results and Discussion}

A total of 60 specimens were collected from patients suspected of Otomycosis based on clinical features. Fungal isolates were found in $44(73.33 \%)$ specimens. All specimens yielded single organism.18 (40.90\%) of Otomycotic patients were males and 26 $(59.10 \%)$ were females.

Aspergillus niger $21 \quad(47.72 \%)$ was the predominant species isolated. Aspergillus flavus $12(27.27 \%)$ was the second most common species isolated. Candida albicans 05 (11.36\%), Aspergillus fumigatus 04 (9.10\%), Mucor 01 (2.27\%) and Penicillium species $01(2.27 \%)$ were the other species isolated (Table 1). Highest frequency of Otomycosis was found in the age group of 15 - 35 years, followed by 36 - 55 years (Table 2).

In our study, among males and females, right ear was predominantly involved and only unilateral involvement of the ear was found. Predominant predisposing factor for Otomycosis was found to be instillation of coconut oil followed by self-cleaning (cleaning of the ear by the patient using unsterile sticks/feather/hair pin) (Table 3). Hearing loss was the predominant symptom in $77.27 \%$ of the Otomycotic patients followed by pain $70.45 \%$. Other symptoms were itching, tinnitus and ear discharge (Table 4).

Otomycosis is a superficial mycotic infection of the external ear canal. Andrall and Gaverret were the first to describe fungal infections of the ear. (Prasad SC et al., 2014). The infection may be either subacute or acute and is 
characterized by pruritus, pain, mild hearing loss, superficial epithelial exfoliation, debris containing fungal hyphae and spores. In the present study isolation rate of fungi from the suspected cases was $73.33 \%$ which is in accordance with other studies (Kaur et al., 2000, Bharti et al., 2011).

Table.1 Mycological profile of otomycosis

\begin{tabular}{|c|l|c|c|}
\hline Sr. No. & \multicolumn{1}{|c|}{ Fungal isolates } & Number of patients & Percentage (\%) \\
\hline 1. & Aspergillus niger & 21 & 47.72 \\
\hline $\mathbf{2 .}$ & Aspergillus flavus & 12 & 27.27 \\
\hline 3. & Candida albicans & 05 & 11.36 \\
\hline $\mathbf{4}$ & Aspergillus fumigatus & 04 & 9.10 \\
\hline $\mathbf{5 .}$ & Mucor Species & 01 & 2.27 \\
\hline 6. & Penicillium Species & 01 & 2.27 \\
\hline
\end{tabular}

Table.2 Distribution of patients according to age group

\begin{tabular}{|c|c|c|c|}
\hline Sr. No. & Age group & Number of Patients & Percentage (\%) \\
\hline $\mathbf{1 .}$ & $5-15$ & 05 & 11.36 \\
\hline $\mathbf{2 .}$ & $16-35$ & 29 & 65.91 \\
\hline $\mathbf{3 .}$ & $36-55$ & 08 & 18.18 \\
\hline $\mathbf{4 .}$ & $>55$ & 02 & 04.55 \\
\hline
\end{tabular}

Table.3 Predisposing factors for otomycosis

\begin{tabular}{|c|l|c|c|}
\hline Sr. No. & Predisposing factors & Number of Patients & Percentage (\%) \\
\hline 1. & Instillation of coconut oil & 16 & 36.36 \\
\hline 2. & Self-cleaning & 14 & 31.82 \\
\hline 3. & No cerumen & 06 & 13.67 \\
\hline 4. & CSOM & 05 & 11.36 \\
\hline $\mathbf{5 .}$ & Prior therapy (Ear drops) & 03 & 06.82 \\
\hline
\end{tabular}

Table.4 Presenting clinical symptoms at the time of diagnosis

\begin{tabular}{|c|l|c|c|}
\hline Sr. No. & Clinical Symptoms & Number of Patients & Percentage (\%) \\
\hline $\mathbf{1 .}$ & Pain & 31 & 70.45 \\
\hline $\mathbf{2 .}$ & Discharge & 14 & 03.10 \\
\hline $\mathbf{3}$ & Itching & 26 & 59.10 \\
\hline $\mathbf{4 .}$ & Hearing loss & 34 & 77.27 \\
\hline $\mathbf{5 .}$ & Tinnitus & 02 & 04.55 \\
\hline
\end{tabular}

Aspergillus spp was found to be predominant fungus isolated from $84.09 \%$ patients followed by Candida and other spp. This finding is in accordance with other findings reported from India (Aneja et al., 2010; Nandyal et al., 2015). Among Aspergillus 
spp, A. niger (47.72\%) was the predominant fungi followed by A. flavus and A. fumigatus, which is similar to other studies (Prasad et al., 2014; Viswanatha et al., 2012). Candida was reported as the predominant organisms with Otomycosis in immunocompromised hosts and in post-operative cavities.

The occurrence of bilateral otomycosis is very low (Yehia et al., 1990). In our study only unilateral involvement was found. Ho et al., observed a bilateral involvement in $7 \%$ of the patients (Ho et al., 2006). Our study population mainly comprised of younger age group which is in accordance with studies from India and other countries. The Incidence of Otomycosis was more in $>15-35$ years age group with $65.91 \%$ cases followed by age group of $>35-55$ years with $18.18 \%$ cases which goes well with other studies (Ho et al., 2006).

Coconut oil has been reported to be sporostatic (Jain et al., 1992) and therefore may help preserve the viability of fungal conidia deposited in the external ear long and indirectly contribute to occurrence of Otomycosis. In our study second most common factor was self-cleaning (31.82\%) (by using unsterile sticks/hair pins etc.) Habit of cleaning ear with such contaminated objects leads to inoculation of fungal debris in external auditory canal. Moreover it damages normal lining epithelium, which is the natural defense that protect against such infections. Other predisposing factors were lack of cerumen, CSOM and prior antibiotic therapy (ear drops). This in accordance with the study conducted by (Pontes et al., 2009).

Among clinical symptoms, Maximum patients presented with hearing loss (77.27\%) followed by pain $70.45 \%$. In another study, pain was reported as the major symptom followed by discharge and hearing loss (Ho et al., 2006).
Rural communities with higher practice of self-cleaning and using home remedies to get relief from ear ailments are at higher risk of Otomycosis. Species like Aspergillus and Candida are the common species involved in such cases. There is a need to educate rural population about serious complications of self-cleaning and using home remedies for ear problems.

\section{References}

Agrawal SR., Jain AK. Goyal RB, Gupta A., Gupta KG. 2001. A clinicomycological study of otomycosis with special reference to silent tympanic membrane perforation. Indian J Otol., 7(2): 49 52.

Aneja KR, Sharma C, Joshi R. 2010. Fungal infection of the ear; a common problem in the north eastern part of Haryana. Int J Ped Otorhinolaryngol., 74(6): 604 607.

Barati B, Okhovvat SAR, Goljanian A, Omrani MR. 2011. Otomycosis in Central Iran: A Clinical and mycological study. Iran Red Crescent Med J.; 13: 873 - 876.

Chander Jagdish, 2018. Textbook of Medical Mycology, $4^{\text {th }}$ Edition. Chandigarh: Jaypee Brothers Medical publishers.

Ho T, Vrabec JT, Yoo D, Coker NJ. 2006. Otomycosis: Clinical features and treatment implications. OtolaryngolHead Neck Surg.; 135: 787 - 791.

Hurst WB. 2001. Outcome of 22 cases of perforated tympanic membrane caused by otomycosis. J Laryngol Otol.; 115: $879-880$.

Kaur R, Mittal N, Kakkar M, Aggarwal AK, Matthur. MD. 2000. Otomycosis: a clinicomycologic study. Ear Nose Throat J.; 79: 606 - 609.

Mishra GS, Mehta N, Pal M. 2004. Chronic bilateral otomycosis caused by Aspergillus Niger. Mycosis; 47: 82 - 84 . 
Nandyal CB, Choudhari AS., Sajjan NB. 2015. A Cross sectional study for Clinico mycological Profile of Otomycosis in North Karnataka. Int $J$ Med Health Sci., 4(1): $64-69$.

Pontes ZB. Silva AD, Lima E, Guerra M, Oliviera N. Carvalho, et al., 2009. Otomycosis: a retrospective study. Braz J. Otortunolaryngol, 75(3): 367 370.

Pradhan B, Tuladhar NR, Amatya RM. 2003. Prevalence of otomycosis in outpatient department of ototaryngology in Tribhuvan University Teaching Hospital, Kathmandu, Nepal. Ann Otol Rhinol Laryngol, 112(4): 384 - 387.

Prasad SC, Kotigadde S, Manisha S et al., 2014. Primary Otomycosis in the Indian
Subcontinent: Predisposing Factors, Microbiology and Classification. International $J$ of Microbiology; Available from: http://dx.doi.org/10.1155/2014/636493

S. K. Jain and S. C. Agrawal. 1992. "Sporostatic effect of some oils against fungi causing otomycosis," Indian Journal of Medical Sciences. 46: 1 - 6.

Viswanatha B., Sumatha D., Vijayashree MS. 2012. Otomycosis in immunocompetent and immunocompromised patients: comparative study and literature review. Ear Nose Throat J., 91:114 - 121.

Yehia MM, AI-Habib HM, Shehab NM. 1990. Otomycosis: a common problem in North Iraq. J Laryngol Otol., 104(5): $387-389$.

\section{How to cite this article:}

Wadile Rahul Gopichand. 2019. Mycological Profile of Otomycosis in Tertiary Care Teaching Hospital. Int.J.Curr.Microbiol.App.Sci. 8(07): 1211-1216. doi: https://doi.org/10.20546/ijcmas.2019.807.144 